\title{
Organization of work in interaction between the paramedics and the patient
}

\author{
Petra Auvinen, ${ }^{\mathrm{a}, *}$ and Hannele Palukka ${ }^{\mathrm{b}}$ \\ ${ }^{a}$ School of Social Sciences and Humanities, Akerlundinkatu 5,33014 University of Tampere, Finland \\ ${ }^{\mathrm{b}}$ School of Management, University of Tampere, Kanslerinrinne 1, 33014 University of Tampere, Finland
}

\begin{abstract}
The paper analyzes the ways in which the two paramedics organize their work activities in the courses of interaction with the patient. The paramedic-patient -interaction is generally based on question-answer-sequences where one paramedic asks a question to which the patient answers. The paper examines the organization of paramedics' institutional roles and responsibilities within these particular sequences of talk by conversation analytic means. Instead of leaning on any personal or psychological explanations, the paper aims to demonstrate how particular breaches or problems in the organization of division of labour become explainable and understandable through the detailed analysis of preceding interaction. The main research data consist of the videorecordings of naturally occurring interactions between paramedics and patient.
\end{abstract}

Keywords: paramedic-patient -interaction, division of labour, question-answer-sequence, conversation analysis, workplace studies

\section{Introduction}

The paramedics are healthcare professionals trained to handle various medical emergencies including car accidents, heart attacks, slips and falls, childbirth and gunshot wounds. The paramedics are often first to arrive at the scene. Their task is to provide emergency care to the patient and, if necessary, transport the patient to a medical facility. The paramedics work in two-person teams in which each member has preassigned duties. Most basically, one paramedic interviews the patient by asking relevant questions while the other conducts the physical examination and drives the ambulance.

This research paper concerns the ways in which the paramedic team maintains their roles and responsibilities in the courses of interaction with the patient. The aim is to describe the organization of division of labour between paramedics through the detailed analysis of interaction. The interaction analysis bases on so called question-answer-sequences, where one paramedic asks the patient questions to which the patient responds. At this point, the issue of who is asking and in what kind of interactional environment asking is done becomes relevant.

In addition to pre-hospital medical care, asking questions is an essential work practice in a wide range of other institutional settings. The studies concerning the activity of questioning and answering are conducted, for example, in the courtroom [2], medical practice $[4,10,12,18]$ and broadcast settings [5].

\section{Theory and methodology}

The paper has its theoretical roots in the anthropology of science and technology and workplace studies $[6,8,19]$. These traditions form a naturalistic approach committed to the detailed study of social and work practices in complex organizational settings. The analytic focus is on the tacit body of reasoning and

\footnotetext{
${ }^{*}$ Corresponding author. E-mail: petra.auvinen@uta.fi
} 
procedures through which the participants produce, make sense of and coordinate activities with each other. Drawing on the combination of the methods of ethnomethodology (EM), conversation analysis (CA) and ethnography, the approach analyzes the production and coordination of tasks in real-time interaction through talk and visual conduct.

CA studies 'talk-in-interaction' [15] - the term encompassing talk and other interactional activity such as physical activities, gestures and paralinguistic features of talk. CA concerns the ways in which utterances accomplish particular social actions in terms of their placement and participation within sequences of action. The primary units of analysis are thus sequences and their component unit turns conceived as turns-within-sequences [9].

The sequences, or, 'courses of action implemented through talk' [16] are generally organized around a basic unit of sequence construction called the 'adjacency pair' [17]. The adjacency pair is a sequence of two utterances being ordered as a first pair part (FPP) and a second pair part (SPP). The production of an utterance identifiable as a FPP (e.g. a question) selects a next speaker who should immediately proceed to produce the appropriate SPP (i.e. an answer). The regular occurrence of certain paired actions is explained by the property of 'conditional relevance' [14], which stipulates that the production of the FPP makes a corresponding SPP both relevant and expectable. This constraint allows the speakers, and the analysts, to recognize whether some conversational events, such as answers to questions, are noticeably absent [9]. Questions and answers are one instance of the first and second parts of the adjacency pair. Others include requests and grants or rejections, invitations and acceptances or refusals, and so on.

The organization of adjacency pair is significant in respect of how intersubjectivity - the mutual understanding of ongoing talk and action - is accomplished and displayed in talk $[9,17]$. As mentioned, the production of a FPP, such as 'invitation', makes relevant a particular action to be done next, or a limited set of such actions. By 'acceptance', among other alternatives, the second speaker not only complies with the adjacency pair structure but also displays an understanding of what the prior utterance was doing [13]. The producer of the initial turn may comment on or correct the second speaker's understanding in the third turn of the sequence. The adjacent positioning enables coparticipants to display their understandings of the ongoing talk and to recognize possible misunderstandings in conversation [17].

\section{Teamwork in pre-hospital medical care}

The paramedic team is typically dispatched by the Emergency Response Centre (ERC) operator to the scene. The ERC operator receives and evaluates the emergency calls from 112 (911 in the U.S.) and hands the assignment over to the appropriate units. The number of units alerted varies depending on the nature and severity of the emergency situation and on unit availability. Providing the patient has, for example, slipped on the street and hurt the knee, the paramedic team can be the only unit dispatched. In major accidents with multiple patients, on the contrary, there might be police, fire fighters and emergency physician working in collaboration with the several paramedic teams. Once the paramedics arrive to the scene, they normally assess the patient's condition and determine his or her medical background. Following protocols and guidelines, they provide emergency care and, if necessary, transport the patient to a medical facility. At the medical facility, the paramedics report their observations and actions to emergency department staff and help transfer the patient to the emergency department.

In paramedic teams, one member generally has a higher level of experience than the other. The paramedics work in the roles of 'attendant' and 'driver' which change from one call to another. For example, the less experienced paramedic may work as an attendant in the first assignment of the shift and as a driver in the next. The driver's task is to drive the ambulance to and from the emergency call and to conduct the physical examination of the patient. The examination typically includes the measurement of the patient's vital signs, such as body temperature, heart rate, breathing rate and blood pressure. Depending on the patient's situation, the driver uses different tools and technologies in patient examination, ranging from a thermometer to a complex defibrillator with 
electrocardiogram (EKG) capability. The driver usually takes care of medication. For example, if a patient is experiencing a significant pain, the driver can administer oral or intravenous drugs in order to stabilize the patient.

The paramedic working as an attendant is responsible for interviewing the patient and documenting the necessary information. The patient interview largely follows the structure and content of a Patient Care Report (PCR) form on which the attendant writes down the information about the patient's current condition and symptoms, his or her present and past illnesses and injuries, allergies, medications, etc. S/he may also consult the physician-on-duty via phone about the patient's treatment. Within paramedic team, the attendant has the authority to lead the conduct of emergency care and make treatment decisions under their responsibilities. Finally, during the transport of the patient, the attendant is seated in the back of the ambulance monitoring the patient's condition and giving additional care.

\subsection{Question-answer-sequences in pre-hospital emergency care}

In patient interview, to put it simply, the paramedic asks questions and patient answers them. One institutional function of questioning in pre-hospital emergency care is to gather necessary information from the patient. The information solicited through these question-answer -sequences is used as a tool in the accomplishment of specific tasks within the overall activity of pre-hospital emergency care, including planning and performing the proper treatment and deciding weather to transport the patient to a medical facility. In the same vain, while the patient provides information to the paramedics through responses, s/he simultaneously displays an understanding of the ongoing task and the opportunities and constraints it offers [4].

The paramedics produce and make their orientation to the patient visible through question-answer sequences. By asking questions from the patients, consequently, the paramedics establish and manage social relationship with them. A caring and trusting relationship with the patient is important in terms of success of emergency treatment. As an example, whether paramedic designs so called closed-ended or open-ended questions in patient interview can have an influence on the quality of interaction and health promotion.

Through the activity of asking the patients questions, the paramedics also establish and render their institutional roles and responsibilities visible. In general outline, the attendant's task is to perform the patient interview while the driver conducts the physical examination at the scene of accident or incident. It is important to note that the division of labour between paramedics is not fixed but is flexible in the sense that the driver is also allowed to ask the patient questions connected to the physical examination.

\subsection{Research data}

The primary research data includes video recordings of interactions between paramedics and patients at the scene and during ambulance transports. A total of twenty-seven (27) ambulance calls were recorded within the Pirkanmaa Hospital District's area in Finland. The paramedic teams participating in the recordings work in Advanced Life Support (ALS) ambulances in either public or private sector. They are relatively highly trained professionals being able to perform such emergency care skills as cardioversion, endotracheal intubation and medication administration. The secondary data consist of ethnographic field observations of work activities in pre-hospital emergency care and eight (8) interviews of paramedic teams.

\subsection{Empirical analysis}

The empirical analysis aims to reveal the ways in which the two paramedics maintain their institutional roles and responsibilities in their interactions with the patient. The analysis of the videotaped interactions centers on the question-answer-sequences in which a paramedic asks a question from the patient. The organization of division of labour between paramedics is examined by analyzing which paramedic is 'doing the asking' and in what kind of sequential environment.

The transcripts shown above describe an emergency care situation taking place in a middle-aged male 
patient's living room. The patient ( $\mathrm{P}$ on the transcripts) having a stomachache is lying on his sofa in a side position. The attendant (PM1 on the transcript) is sitting on another sofa, interviewing the patient and filling out the PCR form. The driver (PM2 on the transcript) is examining the patient with different medical equipment in a standing position. The Emergency Response Centre has determined the urgency category of this emergency call as $\mathrm{C}$ referring to the call with a low risk of being life threatening in the next thirty (30) minutes. The transcription conventions are described before References.

The transcripts 1(a) and 1(b) are shown to reveal how the paramedics adhere to the institutional norms of conduct in their interactions with the patient. In transcript 1(a), the PM1 asks questions as part of the larger activity of patient interview (see lines in bold).

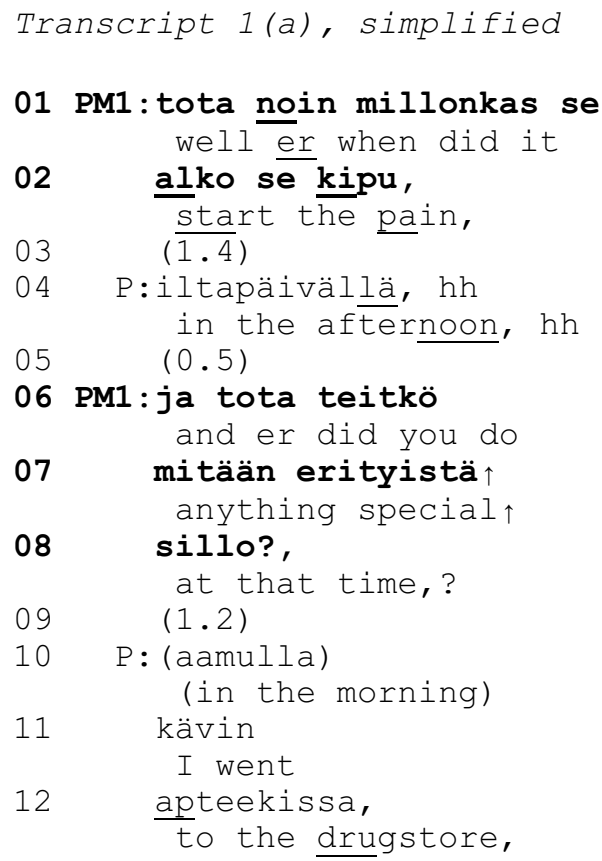

At lines 1-2, the PM1 inquiries the patient about the timing of the onset of pain. Following the patient's response 'in the afternoon', the PM1 delivers andprefaced question [11] at lines 6-8. And-prefacing links the question to the preceding question-answer sequence. It also highlights a routine or agenda-based character of the question it prefaces. With his and- prefaced question, consequently, the PM1 displays an orientation to the common task of patient interview implemented through a series of question-answer pairs. The patient responds by explicating what he had done not in the afternoon, but in the morning when he 'went to the drugstore' (lines 10-12).

In transcript 1(b), it is the PM2 who does the questioning. Here, the production of question by him is socially and institutionally acceptable as it occurs during, and is related to, the physical examination of the patient (see lines in bold).

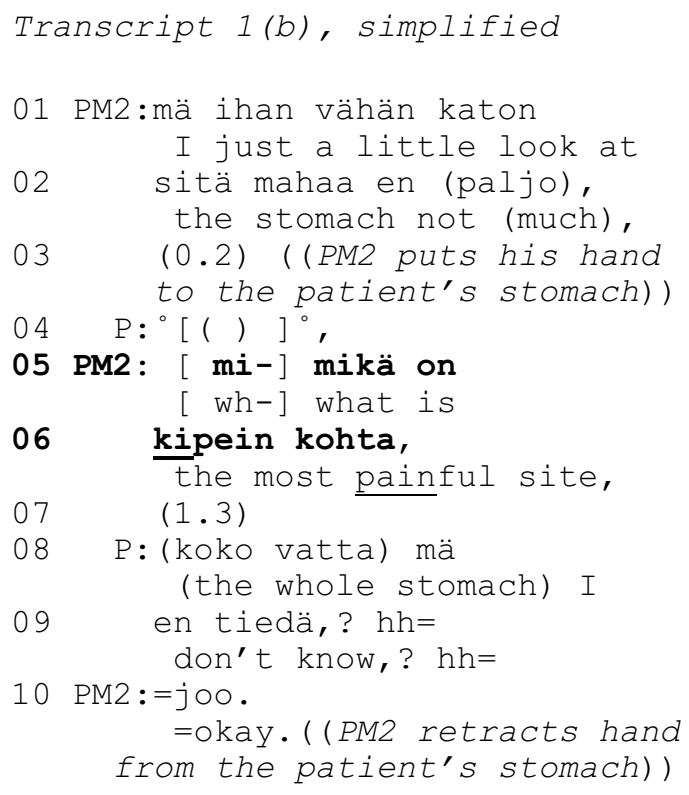

The PM2's talk at lines 1-3 projects his upcoming activity of examining the patient's stomach. The PM2 asks the patient about the location of the highest pain while keeping his hand on the stomach (lines 3-6). The patient gives an ambiguous answer in which he can not fully specify the most painful site (lines 8-9). The PM2 acknowledges the patient's response in the third turn relative to question-answer -sequence ('okay') and completes the physical examination by retracting his hand from the patient's stomach (line 10).

In the final transcript (2), on the contrary, the practical division of labour between paramedics is breached in and through the PM2's questioning activity (see lines in bold). 


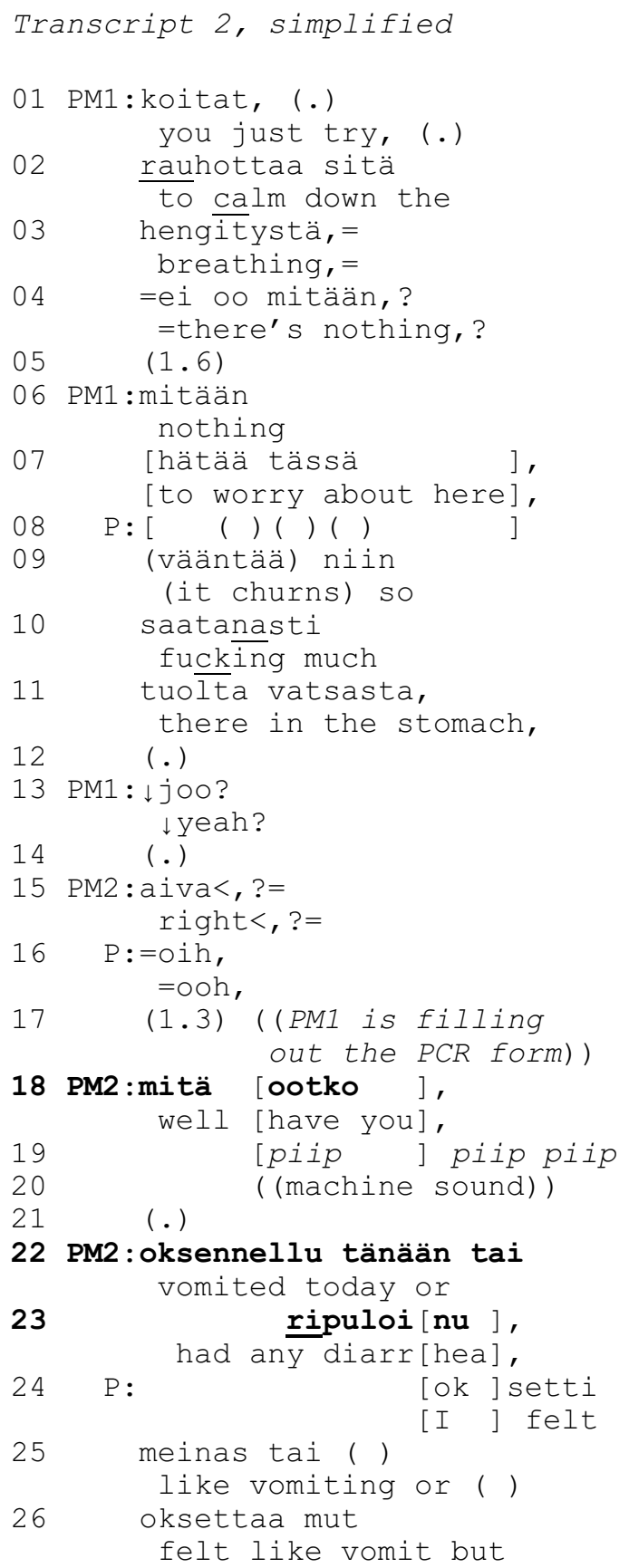

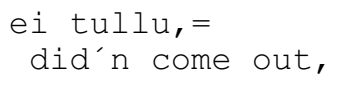

The PM2 steps into the PM1's territory by delivering a question about the symptoms of the pain (lines 18 and 22-23). The aim is to explicate through the detailed analysis of interaction how the PM2 ended up asking that question from the patient in that sequential position. The analytic attention must be therefore directed to the interactive events taking place prior to questioning.

The PM1 advises the patient to 'calm down the breathing' and ensures that 'there's nothing...nothing to worry about' (lines 1-7). The patient seems to implicitly disagree with the PM1 by emphasizing the high intensity of the pain at lines 8-11. Not only the PM1 but also the PM2 respond to the patient's description of pain: the PM1 with the minimal ' $\downarrow$ yeah?' and the PM2 with a slightly more emphatic 'right $<$ ?,'. Before the initiation of the PM2's questioning, the PM1 is oriented to the non-verbal activity of filling out the PCR form (lines 17-18); simultaneously with the initiation of the question, there occurs a three-part beeping sound (lines 18-20). The sound indicates the defibrillator machine has finished processing the patient's blood pressure data. After the sound of machine, the PM2 completes his questioning about the symptoms of pain (lines 19-23); the patient responds to the question at lines 24-27.

The PM2's question takes place in an interactive environment where the PM1 is engaged not in talking with the patient but in writing activity. The question is also initiated while the defibrillator machine is processing the patient data. In this way, while waiting for the data processing to be finished, the PM2 is available for interaction with the patient. The PM2 is not just an over-hearer of an ongoing talk but actively participates in it by responding the patient's description of pain even though the PM1 had already done so.

To conclude, the occurrence of the PM2's question in that particular sequential position can be explained through the preceding interaction. In this case, then, the norms of interaction in a way override the institutional norms of conduct. After talking with the patient, the PM2 announces the blood pressure values to the PM1 (not shown on the transcript). The PM1 writes the values down and continues asking the patient questions 
(see Transcript 1(a)). Within a minute's time, the PM1 partly repeats the PM2's question by asking the patient about the functioning of stomach and intestine.

\section{Conclusions}

Asking questions from the patient is a central work practice of pre-hospital emergency care. The paramedics use questions to gather information about the patient in order to get the job done. Through questions and answers, the paramedics and patients also collaboratively create and maintain social relationships with each other. Moreover, the paramedics produce and make their mutual roles and responsibilities visible through questioning.

This paper concerns the relation between the practice of questioning and division of labour in pre-hospital emergency care. As our previous research on aviation interaction $[1,3]$, the paper integrates the detailed analysis of the sequences of talk into the examination of the larger sequential organization of action. Theoretically, therefore, the paper sheds light to the close relationship between talk and action by showing that these two modalities are not separated but are juxtaposed in ways which mutually elaborate each other [7].

The paramedic team orients to the patient and to their institutional duties as an 'attendant' and 'driver' through talk and visual conduct. This kind of a doubleorientation appears problematic in cases where one paramedic asks questions from the patient despite the fact that it is not his responsibility to do so. In this paper, these breakdowns of paramedical roles and responsibilities are not explained by the personality traits of the paramedics or by the personal chemistry between the two. They are instead explicated by the preceding courses of interaction between the paramedic(s) and the patient.

The systematic analysis of how division of labour between paramedics is organized in their interactions with the patient continues. More data needs to be analyzed in order to make inferences about the phenomenon. Especially the breaches of paramedics' roles and responsibilities will be analyzed further (e.g., their frequency of occurrence in data, how the paramedics deal with them and what kind of consequences they have for interaction and conduct of emergency care).

\subsection{Transcription conventions}

[ ] Interlocking left-brackets indicate where overlapping talk begins; interlocking right-brackets indicate where overlapping talk ends.

$=$ Equal signs, one at the end of one line and one at the beginning of a next, indicate no "gap" between the two lines.

(1.5) Silence measured in seconds and tenths of seconds.

( . ) Silence of less than a fifth of a second, i.e., less than (0.2).

He says Underscoring indicates some form of stress, via pitch and/or amplitude.

$\uparrow \downarrow \quad$ Arrows indicate shifts into higher or lower pitch.

., ? ,? Punctuation markers are used to indicate intonation.

- $\quad$ The degree sign is used as a "softener." Utterances or utterance parts bracketed by degree signs are relatively quieter than the surrounding talk. < A pre-positioned left carat indicates a "hurried start." A post-positioned left carat indicates a "sudden stop." .hhh A dot-prefixed row of hs indicates an in-breath. Without the dot the hs indicate the out-breath.

( ) Empty parentheses indicate the transcriber's inability to hear what was said. The amount of the parenthesized space indicates the length of the untranscribed talk.

(word) Parenthesized words are especially dubious hearings or speaker-identifications.

(( )) Doubled parentheses contain transcriber's comments.

(()) Doubled parentheses (italic) contain the description of non-talk activity.

\section{References}

[1] I. Arminen, P. Auvinen and H. Palukka, Repairs as the last orderly provided defense of safety in aviation, Journal of Pragmatics 42 (2010), 443-465.

[2] J.M. Atkinson and P. Drew, Order in Court: The Organization of Verbal Interaction in Judicial Settings, Macmillan, London, 1979.

[3] P. Auvinen, Achievement of Intersubjectivity in Airline Cockpit Interaction, Ph.D. Dissertation, University of Tampere, 2009. 
[4] E. Boyd and J. Heritage, Taking the history: questioning during comprehensive history-taking, in: Communication in Medical Care. Interaction between Primary Care Physicians and Patients, J. Heritage and D.W. Maynard, eds., Cambridge University Press, Cambridge, 2006, pp. 151-184.

[5] S. Clayman and J. Heritage, The News Interview. Journalists and Public Figures on the Air, Cambridge University Press, Cambridge, 2002.

[6] C. Goodwin, Seeing in Depth, Social Studies of Science 25 (1995), 237-274.

[7] C. Goodwin, Action and embodiment within situated human interaction, Journal of Pragmatics 32 (2000), 14891522 .

[8] C. Heath and P. Luff, Technology in Action, Cambridge University Press, Cambridge, 2000.

[9] J. Heritage, Garfinkel and Ethnomethodology, Polity Press, Cambridge, 1984

[10] J. Heritage, Questioning in Medicine, in: Why do you ask?: The Function of Questions in Institutional Discourse, A. Freed and S. Ehrlich, eds., Oxford University Press, New York, 2010, pp. 42-68.

[11] J. Heritage and M-L. Sorjonen, Constituting and maintaining activities across sequences: And-prefacing as a feature of question design, Language in Society 23 (1994), 1-29.

[12] J.D. Robinson and J. Heritage, Physicians' opening questions and patients' satisfaction, Patient Education and Counseling 60 (2006), 279-285.

[13] H. Sacks, E.A. Schegloff and G. Jefferson, A Simplest Systematics for the Organization of Turn-Taking for Conversation, Language 50 (1974), 696-735.

[14] E.A. Schegloff, Sequencing in Conversational Openings, in: Directions in Sociolinguistics. The Ethnography of Communication, J.J. Gumperz and D. Hymes, eds., Holt, Rinehart and Winston, New York, 1972, pp. 346-380.

[15] E.A. Schegloff, Between Macro and Micro: Contexts and other connections, in: The Macro-Micro Link, J. Alexander, B. Giessen, R. Munch and N. Smelser, eds., University of California Press, Berkeley, 1987, pp. 207234.

[16] E.A. Schegloff, Sequence Organization in Interaction. A Primer in Conversation Analysis 1, Cambridge University Press, Cambridge, 2007.

[17] E.A. Schegloff and H. Sacks, Opening up Closings, Semiotica 8 (1973), 289-327.

[18] T. Stivers and J. Heritage, Breaking the sequential mold: Answering 'more than the question' during comprehensive history taking, Text 21 (2001), 151-185.

[19] L. Suchman, Plans and Situated Actions: the problem of human-machine communication, Cambridge University Press, Cambridge, 1987. 